\title{
Looking toward the Future: An Inquiry into the Acceptance of a Hypothetical Laboratory-based Screening Test for Autism Spectrum Disorder
}

\author{
Jane M Deluca, PhD, RN, CPNP-PC ${ }^{1 *}$, Sara M Sarasua, PhD, MSPH ${ }^{1}$ and Luigi Boccuto, MD \\ ${ }^{1}$ Clemson University School of Nursing, USA \\ ${ }^{2}$ The Greenwood Genetic Center, USA
}

*Corresponding author: Jane M DeLuca, PhD RN CPNP-PC, Clemson University School of Nursing, 421 Edwards Hall, Box 159, Clemson, South Carolina 29634, USA, Tel: 864-656-5522, Fax: 864-656-5488

\begin{abstract}
Background: Currently, a clinical diagnosis of autism spectrum disorder (ASD) is determined by behavioral/ observational evaluations performed on children ages 2-5 years. Research suggests that laboratory-based tests for screening or diagnosis of ASD may be available in the future. In this study, we examined attitudes towards the acceptance of a hypothetical laboratory-based screening test for children for ASD.

Methods: For this study, a survey was developed to capture opinions about a hypothetical ASD laboratory test and issues related to screening program functions such as costs of screening. Sixty-five people participated in the study.

Results: The majority of participants would consider screening a child for ASD with a laboratory-based blood test if available. Most approved of early screening before age three regardless of the presence of ASD symptoms. If a screening test indicating a risk of developing ASD, most would consider enrolling a child in an early intervention program.

Discussion and conclusions: The trends in this preliminary work indicate favorable attitudes toward an early-initiated laboratory-based screening test for ASD. In advance of a clinically available test for ASD, understanding people's responses to such screening can help us conceptualize strategies for counseling and care delivery.
\end{abstract}

\section{Keywords}

Autism, Newborn screening, Family, Biomarkers, ASD, Ambiguity, Screening programs

\begin{abstract}
Abbreviations
NBS: Newborn Screening; ASD: Autism Spectrum Disorder; AAP: The American Academy of Pediatrics; M-CHAT-R/F: Modified Checklist of Autism in Toddlers, Revised; TFA: Tolerance for Ambiguity Scale; SPSS: Statistical Package for the Social Sciences
\end{abstract}

\section{Introduction}

The past decade has brought rapid growth in genomic sequencing technology, metabolomic screening, and diagnostic testing. Interest in applying genomic sequencing to newborn screening (NBS) prompted the National Institutes of Health to award several grants in this area [1]. Metabolomics has also shown promise in the identification of biomarkers for a number of conditions, including autism spectrum disorder (ASD) [2]. Advances in biomedical screening techniques may someday enable pre-symptomatic identification of children at increased risk for ASD and developmental disorders. In practice, screening programs are successful when predicated on public acceptance of genetic or biomarker testing if early diagnosis can alter the phenotype.

Autism spectrum disorder (ASD) constitutes a group of neuro developmental conditions featuring three primary symptoms: impaired social interactions, limitations in communication abilities, and repetitive behaviors. Additional features of ASD may include intellectual disabilities, hypotonia, gastrointestinal issues, and sei-

Citation: DeLuca JM, Sarasua SM, Boccuto L (2019) Looking toward the Future: An Inquiry into the Acceptance of a Hypothetical Laboratory-based Screening Test for Autism Spectrum Disorder. Int J Pediatr Res 5:050. doi.org/10.23937/2469-5769/1510050

Accepted: February 21, 2019: Published: February 23, 2019

Copyright: (C) 2019 DeLuca JM, et al. This is an open-access article distributed under the terms of the Creative Commons Attribution License, which permits unrestricted use, distribution, and reproduction in any medium, provided the original author and source are credited. 
zures [3]. ASD is high in the U. S. with a prevalence of 1 in 59 for children age eight according to the Centers for Disease Control and the Autism and Developmental Disabilities Monitoring (ADDM) Network [4]. Evidence supporting a genetic predisposition for ASD is seen in high concordance rates for identical twins (80-90\%), sibling recurrence $(10-30 \%)$, and gene changes in $15 \%$ to $40 \%$ of children with ASD $[1,5,6]$. However, there are hundreds of gene variants associated with the condition [7]. Whole exome studies suggest possibilities for identifying pathogenic mutations in patients with clinical features of ASD [8]. Select biomarkers that have associated with ASD include elevated serotonin levels in platelets $[9,10]$, abnormal urinary catabolites $[11,12]$, oxidative biomarkers [13], serum metabolites [14], and abnormal tryptophan metabolism [15].

ASD is currently diagnosed through clinical assessments and observations of children provided by pediatric healthcare providers, families, and teachers. Autism can be ascertained in children as young as two years of age [16]. Multidisciplinary assessments and clinical tools for diagnosing ASD appear most accurate when applied around the age of 3-5 years, though tools are in development to identify younger children with ASD $[17,18]$. The importance of early diagnosis lies in initiating early treatment. There is evidence that early intervention has been helpful for improving language skills, adaptive behaviors (social, motor, and daily living), and in reducing severity of ASD symptoms [19-21]. The American Academy of Pediatrics (AAP) recommends screening of children by 18 to 24 months of age with instruments such as the Modified Checklist of Autism in Toddlers, Revised, (M-CHAT-R/F) [22-24]. However, routine clinical screening is not universally adopted, potentially missing at-risk children [21,25,26]. Studies indicate that children among specific ethnic groups may be under diagnosed $[27,28]$. For these reasons, early ASD screening based a valid laboratory test would be highly useful in identifying at-risk children for early institution of developmental services.

In anticipation of the development of a potential laboratory-based screening test for ASD, we conducted a preliminary investigation into public interest for a "hypothetical" laboratory-based test for ASD. We sought to understand people's perspectives of early childhood screening for ASD by a laboratory-based test. We queried study participants for their opinions of the implementation of early intervention services for children at high risk for ASD and their preferences for the delivery of ASD screening services. Because initial screening can yield ambiguous results, and there may be considerable wait time for ASD symptoms to be clinically observable (or not, in the case of false-positive results). We asked participants' for their perspectives on the uncertainties of results and a presumed time gap between an early lab-based screening test and age of onset of clinical symptoms for determining a definitive diagnosis.

\section{Methods}

\section{Newborn screening as a model for an ASD screening program}

For this pilot study, we developed a survey for investigating peoples' views of a hypothetical laboratory-based ASD screening test for children. NBS is a premiere screening program for identifying many disorders in infancy, providing early treatment, and ultimately improving outcomes for children. Complex conditions such as metabolic disorders, cystic fibrosis, and congenital heart disease can be identified through NBS. NBS offers important lessons to consider in developing and executing screening programs. We used aspects of NBS programs to develop our survey questions for our hypothetical laboratory-based screening program for ASD. We identified four key areas to focus our inquiry. These included 1) Participant acceptance and uptake of a hypothetical ASD laboratory screening test; 2) Participant responses to screen-positive, ambiguous, or false-positive results; 3) Preferences for the administration of developmental therapies for children at risk for ASD; preferences and practical considerations for the delivery of screening results and screening costs, and 4) Assessing barriers to accessing developmental therapies. In addition, we queried participants' interest in participating in ASD research.

We administered an initial version of our survey instrument to ten parents of children with (3/10) and without autism (7/10), this data was not included in the study. The group suggested improvements for the clarity of the questions and the content, which were incorporated into the survey. The finalized survey was a 20-item instrument that included the 7-question TFA scale. It took approximately 10 minutes for study participants to complete.

\section{Ethical approval and recruitment procedures}

Two institutional review boards approved the study protocol. Consent was obtained from all participants for their inclusion in the study. Demographic and survey data was collected anonymously from participants. The survey was offered to parents and family members (18 years and older) waiting in pediatric offices for appointments for their children. Two pediatric practice locations served as recruiting sites for a convenience sample of participants. The data collection took place from 2014 to 2015.

\section{Statistical analyses}

Descriptive statistics were used to calculate frequencies in the Statistical Package for the Social Sciences (SPSS) software version 22 for each demographic category and survey question [29]. 


\section{Results}

\section{Participant demographics}

Sixty-five participants completed the survey; $93 \%$ were female. High proportions were white (81.3\%). Most had middle or high levels of education at $65.7 \%$ with at least some years of college or college degrees. The majority was employed (63.1\%), and most were at mid-lower income levels $(70.3 \%,<\$ 39,999$ US dollars per year). The mean number of children per participant was 2.2. Six participants claimed no biological children. The mean age of participants was 36.1 (range 26-67 years). Four of 65 participants had family members diagnosed with ASD; two had children with ASD and two reported another family member (not specified) with an ASD diagnosis.

\section{Willingness to take a blood test indicating a risk for ASD}

The majority of respondents $(44 / 65,69 \%)$ indicated they were willing to have their child "take a blood test that could indicate a risk of developing ASD" (Table 1 , Q1). Slightly fewer $37 / 62$ (59\%) indicated they were willing to have their child, "take a blood test that could indicate a risk of developing ASD, even if the test result will not guarantee with absolute certainty an ASD diagnosis". All four participants with family members with ASD reported willingness to test a child, even if the test was not diagnostic and only indicated risk of ASD (Table 1, Q2).

With the lag in time between the early-administered ASD laboratory-based screening test and definitive clinical assessment at around age 3, participants were evenly divided between wanting to pursue testing only in the presence of signs of ASD (51\%) or without signs of ASD (49\%). Regardless of the presence of signs of ASD or not, most expressed a preference for testing as early as possible $(83 \%)$ rather than waiting until age $3(17 \%)$ (Table 1, Q3).

In the hypothetical case of a child testing "at-risk" for ASD, the majority (75\%) of participants reported that with screen-positive ASD results, even without

Table 1: Select survey questions and results.

\section{Question}

Response

Q1. Would you be willing to have your child take a blood test that Yes

could indicate a risk of developing ASD?

No

Q2. Would you be willing to have your child take a blood test that Yes could indicate a risk of developing ASD, even if the test result will No not guarantee with absolute certainty an ASD diagnosis? (i.e., your child could be called "at risk" of developing ASD, but would never actually develop any behavioral problems or ASD).

Q3. Currently, the clinical diagnosis of ASD cannot be made until the age of 3 , while the screening blood test for ASD, although not diagnostic, seems to be reliable before the age of 3 . Considering the possibility of a time gap between the screening test and the age for a definitive diagnosis, would you rather have your child:

Q4. You decide to have your child tested even if he/she does not show any behavioral problems. The result you receive suggests a risk for ASD for your child. How would you mainly feel about this?

a. Tested as early as possible, even without signs suggestive of ASD

b. Tested as early as possible only in the presence of signs suggestive of ASD

c. Tested at age close to 3 years, 1 even without signs suggestive of ASD

d. Tested at age close to 3 years, 9 only in the presence of signs suggestive of ASD

a. Glad, because the test will help 44

\begin{tabular}{|l|l|}
\hline Frequency & Percent \\
\hline 44 & $69 \%$ \\
\hline 21 & $21 \%$ \\
\hline 37 & $59 \%$ \\
\hline 26 & $41 \%$ \\
\hline
\end{tabular}

identify a potential disorder and I can help my child

b. Distressed, because of the 7 potential challenges of raising a child with an ASD

c. Scared, because I would not know what to do

d. Upset, because ASD would affect the life of the entire family

Q5. If your child had a laboratory screening test indicating a a. Yes, since early intervention high risk of developing ASD, would you enroll him/her in an early programs do not cause harmful intervention program?

side effects and might help

b. Yes, but only if the child exhibits signs suggestive of ASD

c. No, only children with a clinical 4

$27 \quad 47 \%$
diagnosis of ASD should be enrolled 
the presence of ASD symptoms, they would feel "Glad, because the test will help to identify a potential disorder and I can help my child" (Table 1, Q4). Those indicating an unwillingness to have their child screened reported slightly higher percentages of anticipated negative feelings if the screening test suggested a potential ASD diagnosis (Figure 1a).

\section{Enrolling in early intervention services}

If the hypothetical screening test indicated a risk of developing ASD, $73 \%$ of participants were willing to enroll their presymptomatic child in an early intervention program. An additional $21 \%$ were willing to enroll their child if symptoms of ASD were present (Table 1, Q5). Cost of early intervention therapies were an anticipated barrier to enrolling in an early intervention program according to $80 \%$ of participants. However, those choosing not to have their child screened were slightly more likely to report additional anticipated barriers to early intervention participation (Figure 1b). Both the test/ not test groups expected traveling to therapies to be a barrier while having a therapist come to the home as more convenient. Participants who were willing to have a child screened for ASD were less likely to view participation in developmental therapies as negative or as a source of stigma for a child (Figure 1b).

\section{Reporting results and costs of the screening test}

When asked with whom participants would share the results of the hypothetical ASD blood screening test, most indicated that they would tell their child's doctor (95\%), with fewer willing to share results with their family members $(87 \%)$, a child's school or teacher $(75 \%)$, or friends $(60 \%)$. The majority of participants preferred to hear about the ASD screening test results in-person from their health care provider (79\%). Only

\section{A: How Would Participant Feel if Child's Test Result Suggests a Risk for ASD by Willingness to Take the Test}

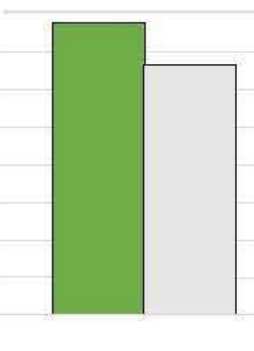

Glad

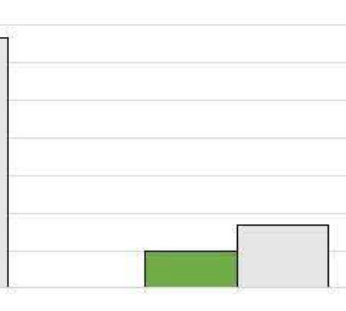

Distressed

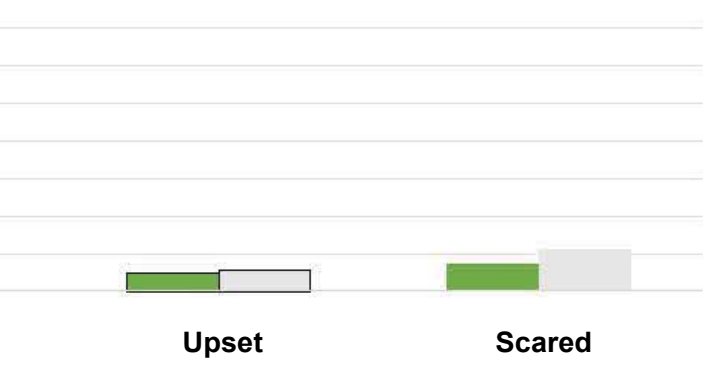

Eest

Not Test

Predominant Feeling

\section{B. Anticipated Barriers to Early Intervention by Willingness to Take the Test}

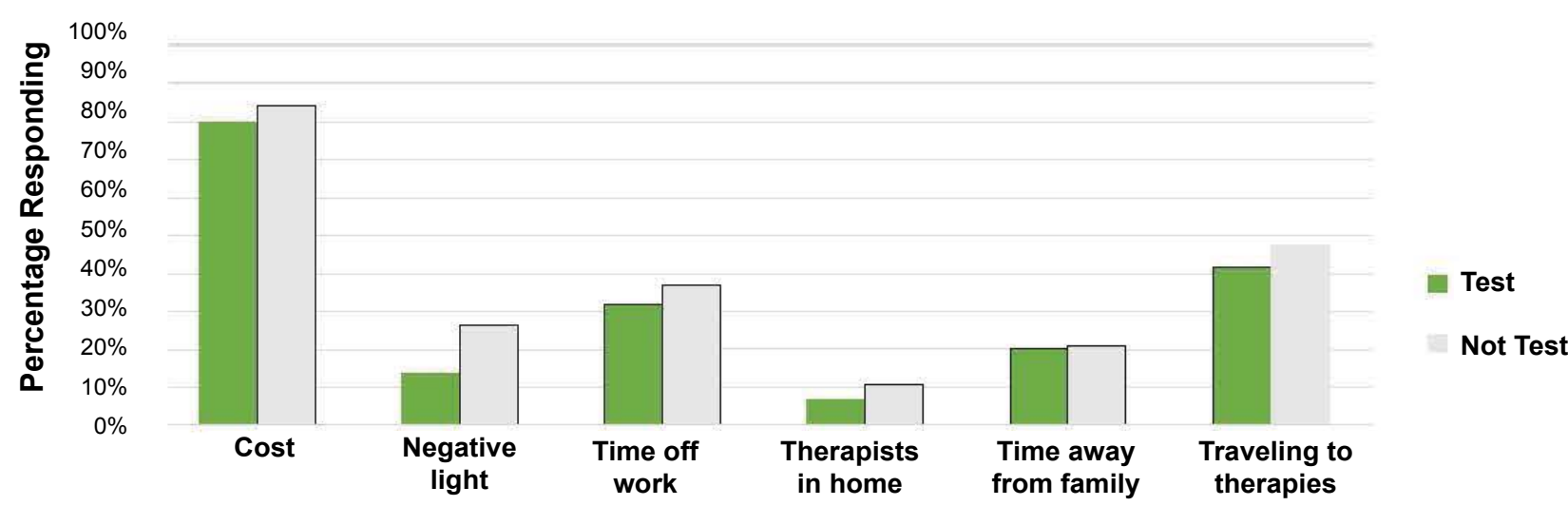

Types of Anticipated Barriers to Enrolling in Early Intervention

Figure 1: a) Participants were asked, "Please imagine the hypothetical case that you decided to have your child tested even if he/she does not show any behavioral problems. The result you received suggests a risk for ASD for your child. How would you mainly fee about this?" We divided participants between who would choose to test versus those who would not. Most were glad of the information, but non-text takers were more distressed, upset or scared; b) Participants were asked the question "Potential barriers to enrolling your child in an early intervention program could include which of the following reasons? (Pick as many as apply):" Non-test takers perceives more barriers to obtaining early intervention services. 
$18 \%$ preferred receiving results by telephone and $3 \%$ preferred notification by mail. The majority of participants preferred a less-than-one-week turnaround time for reporting ASD results (74\%). The vast majority desired the laboratory test to be either free or covered by insurance and costing less than $\$ 50$ US dollars $(87 \%$ of respondents).

\section{Engaging in ASD research}

Most participants, 92\%, were willing to authorize use of de-identified results for ASD research. Roughly, 70\% indicated interest in their families' direct involvement in the development of a laboratory-based test for ASD, if such a study existed, although $11 \%$ would participate only if paid for their time.

\section{Discussion}

The majority of study participants thought the value of an ASD laboratory-based screening test outweighed the risks and discomforts of the test rendering positive, false-positive, or inconclusive results. Previous studies of parental perspectives of genetic testing in autism noted positive attitudes toward genetic screening tests for ASD [30-32]. This study expands our understanding of peoples' responses to screening tests that do not lead to an immediate diagnosis but may take months or years to resolve. The results indicated that participants for the most part were appreciative of the knowledge of increased autism risk that could lead to an opportunity to help a child.

In our study, parents agreed they would accept early intervention services if their child was at risk for ASD. However, it is important to note that study participants admitted to potential barriers such as personal costs and travel to services that could interfere with these interventions. In recent years, there have been notable increases in ASD diagnoses accompanied by greater demands for early intervention services [33,34]. It would be difficult to reconcile costs for instituting early intervention services in presymptomatic cases of screen-positive findings for autism. Ongoing monitoring of children at higher risk raises questions of costs for surveillance and the potential psychological stress for families during this observation period. Providing services to at-risk children before symptoms manifest would require policy changes for possible expansion of early intervention services and the development of efficient models for fair distribution of therapeutic interventions.

In response to the rapid expansion of NBS enabled by advances in screening technology, the American College of Medical Genetics refined NBS inclusion criteria to incorporate primary disease targets that meet standardized screening criteria, and secondary targets identified in pursuit of the primary targets [35]. These guidelines do not address the procedural and ethical concerns rising from issues of risk and uncertainty in screening and genetic testing. As demonstrated in our hypothetical study, screening could include the identification of markers that may confer increased risk but are not in themselves diagnostic. Policies are needed that address these new diagnostic paradigms and help solve the problems of test utility and diagnostic uncertainties in the provision of effective, ethical screening for the health of all children [36,37].

This work was very preliminary in nature. The total number of participants was very small and the sample was relatively homogenous with regard to gender, income, age, ethnicity, and locality. These limitations preclude generalizing these findings to other groups or drawing firm conclusions about the acceptance of a potential screening test. Further refinement of the survey is necessary for validation, and developing additional, nuanced questions about attitudes toward potential screening tests. Although the uptake by participants appeared good, we do not know how many potential participants rejected the survey or how they would have answered the questions differently from those who participated in the study. This work was an exploration of a hypothetical screening test for ASD and we believe the study participants understood the study aims. We do not know if these results would be comparable in practice if an ASD test was actually available.

\section{Conclusions}

While not yet in existence, a laboratory-based screening test for ASD could have significant utility for identifying children at-risk for autism and ameliorate issues associated with the clinical determination of ASD while improving outcomes by providing earlier treatment. Future studies can include increasing the numbers and diversity of study participants for understanding more broadly based opinions in the population about a potential ASD laboratory-based screening test. It would be very important to survey families of children with ASD who are engaged in research of biologically based screening tests to understand their responses to a potential screening test, their preferences for the results reporting process, and types of clinical supports needed for the development of optimal screening programs.

While there are potential gains from screening programs despite uncertainties in testing, there are important caveats to observe. The ethical, legal, and social implications of new genetic or biochemical testing and technologies frequently lag behind technological developments. It is hoped our study raises some of the essential questions to be asked as screening moves to new and varied test platforms. In an ideal world, screening programs offer well-developed screening tests that minimize the uncertainties associated with screen results. This is not always the case in the modern era of screening. Skillful clinical genetic counseling services 
are a necessary component for caring for families to ensure that they have adequate understanding of complex screening results. Provider communication and counseling skills would be critically valuable in the context of a clinical screening test for autism or other complex disorders.

\section{Acknowledgments}

We wish to thank the families who particpated in the study and completed the survey. We would like to thank the cooperating pediatric practices and clinic staff for their assistance with the study.

\section{Funding}

This study was not funded.

\section{Author Contributions}

The authors contributed equally to this study and subsequent manuscript. JMD, SMS and LB were responsible for the study concept and design. JMD planned the research protocol, was responsible for data collection, and assisted in data analysis. SMS performed data/statistical analysis and designed tables and figures. LB developed the literature review, assisted in planned the research protocol and data analysis. All authors were responsible for drafting and writing the article. All authors read and approved the manuscript.

\section{Ethics Approval and Consent to Participate}

The institutional review boards of Clemson University (2014-107), Clemson, South Carolina and Self Regional Healthcare (00005425), Greenwood South Carolina approved the study protocol, consent, and survey instrument. All procedures followed were in accordance with the ethical standards of the responsible committee on human experimentation (institutional and national) and with the Helsinki Declaration of 1975, as revised in 2000. Consent was obtained from all participants in the study. Data was collected anonymously.

\section{Availability of Data and Material}

The data generated and analyzed for this study are included in this manuscript.

\section{Competing Interests}

Jane M. DeLuca, Sara M. Sarasua, and Luigi Boccuto declare they have no conflicts of interest.

\section{References}

1. National Institutes of Health (NIH) (2013) NIH program explores the use of genomic sequencing in newborn healthcare. U.S. Department of Human Services.

2. West PR, Amaral DG, Bais $P$, Smith AM, Englash LA, et al. (2014) Metabolomics as a tool for discovery of biomarkers of autism spectrum disorder in the blood plasma of children. PLoS One 9: e112445.

3. Miles JH (2011) Autism spectrum disorders-a genetics review. Genet Med 13: 278-294.
4. Baio J, Wiggins L, Christensen DL, Maenner MJ, Daniels $\mathrm{J}$, et al. (2018) Prevalence of autism spectrum disorder among children aged 8 years-Autism and developmental disabilities monitoring network, 11 sites, United States, 2014. MMWR Surveill Summ 67: 1-23.

5. Mak CM, Lee HC, Chan AY, Lam CW (2013) Inborn errors of metabolism and expanded newborn screening: Review and update. Crit Rev Clin Lab Sci 50: 142-162.

6. Schaefer GB, Mendelsohn NJ, Professional Practice and Guidelines Committee (2013) Clinical genetics evaluation in identifying the etiology of autism spectrum disorders: 2013 guideline revisions. Genet Med 15: 399-407.

7. Simons Foundation Autism Research Initiative (SFARI) (2016) Human Gene Module. An integrated catalogue of human genetic studies related to autism.

8. O'Roark BJ, Vives L, Girirajan S, Karakoc E, Krumm $\mathrm{N}$, et al. (2012) Sporadic autism exomes reveal a highly interconnected protein network of de novo mutations. Nature 485: 246-250.

9. Anderson GM (2002) Genetics of childhood disorders: XLV. Autism, Part 4: Serotonin in autism. J Am Acad Child and Adolesc Psychiatry 41: 1513-1516.

10. Schain RJ, Freedman DX (1961) Studies on 5-hydroxyindole metabolism in autistic and other mentally retarded children. J Pediatr 58: 315-320.

11. Ming X, Stein TP, Barnes V, Rhodes N, Guo L (2012) Metabolic perturbance in autism spectrum disorders: A metabolomics study. J Proteome Res 11: 5856-5862.

12. Yap IK, Angley M, Veselkov KA, Holmes E, Lindon JC, et al. (2010) Urinary metabolic phenotyping differentiates children with autism from their unaffected siblings and agematched controls. J Proteome Res 9: 2996-3004.

13. Adams JB, Audhya T, McDonough-Means S, Rubin RA, Quia D, et al. (2011) Nutritional and metabolic status of children with autism vs. neurotypical children, and the association of autism severity. Nutr Metab (Lond) 8: 34.

14. Wang $\mathrm{H}$, Liang $\mathrm{S}$, Wang $\mathrm{M}$, Gao J, Sun C, Wang J, et al. (2016) Potential serum biomarkers from a metabolomics study of autism. J Psychiatry and Neurosci 41: 27-37.

15. Boccuto L, Chen CF, Pittman AR, Skinner CD, McCartney $\mathrm{HJ}$, et al. (2013) Decreased tryptophan metabolism in patients with autism spectrum disorder. Mol Autism 13: 16.

16. Lorde C, Risi S, DiLavore PS, Shulman C, Thurm A, et al. (2006) Autism from 2 to 9 years of age. Arch Gen Psychiatry 63: 694-701.

17. Falkmer T, Anderson K, Falkmer M, Horlin C (2013) Diagnostic procedures in autism: A literature review. Eur Child Adolesc Psychiatry 22: 329-340.

18. Towle PO, Patrick PA (2016) Autism spectrum disorder screening instruments for very young children: A systematic review. Autism Res Treat.

19. Dawson G, Rogers S, Munson J, Smith M, Winter J, et al. (2010) Randomized, controlled trial of an intervention for toddlers with autism: The Early Start Denver Model. Pediatrics 125: e17-e23.

20. Reichow B (2012) Overview of meta-analyses on early intensive behavioral intervention for young children with autism spectrum disorders. J Autism Dev Disord 42: 512520.

21. Zwaigenbaum L, Bauman ML, Choueiri R, Kasari C, Carter A, et al. (2015) Early intervention for children with autism 
spectrum disorder under 3 years of age: Recommendations for practice and research. Pediatrics 136: S60-S81.

22. Johnson CP, Myers SM, American Academy of Pediatrics Council on Children with Disabilities (2007) Identification and evaluation of children with autism spectrum disorders. Pediatrics 120: 1183-1215.

23. McPheeters ML, Weitlauf AS, Vehorn A, Taylor C, Sathes NA, et al. (2016) Screening for autism spectrum disorder in young children: A Systematic evidence review for the U.S. Preventive Services Taskforce. Evidence Synthesis No. 129.

24. Robins DL, Casagrande BS, Barton M, Chen CM, DumontMathieu T, et al. (2014) Validation of the modified checklist for autism in toddlers, revised with follow-up (M-CHATR/F). Pediatrics 133: 3745.

25. Stenberg N, Bresnahan M, Gunnes N, Hirtz D, Hornig $M$, et al. (2014) Identifying children with autism spectrum disorder at 18 months in a general population sample. Paediatr Perinat Epidemiol 28: 255-262.

26. Zuckerman KE, Lindly OJ, Sinche BK (2015) Parental concerns, provider responses, and timeliness of autism spectrum disorder diagnosis. J Pediatr 166: 1431.e1-1439.e1.

27. Mandell DS, Wiggins LD, Carpenter LA, Daniels J, DiGuiseppi C, et al. (2009) Racial/Ethnic disparities in the identification of children with autism spectrum disorders. Am J Public Health 99: 493-498.

28. Zuckerman KE, Mattox K, Donelan K, Bathayar O, Baghaee BS, et al. (2013) Pediatrician identification of Latino children at risk for autism spectrum disorder. Pediatrics 132: 445-453.

29. (2013) Statistical Package for the Social Sciences (SPSS) for Windows, Version 22.0. IBM Corp, Armonk, NY.
30. Chen LS, Xu L Huang TY, Dhar SU (2013) Autism genetic testing: A qualitative study of awareness, attitudes, and experiences among parents of children with autism spectrum disorders. Genet Med 15: 274-281.

31. Narcisa V, Discenza M, Vaccari E, Rosen-Sheidley B, Harden AY, et al. (2012) Parental interest in genetic risk assessment test for autism spectrum disorders. Clin Pediatr (Phila) 52: 139-146.

32. Xu L, Mitchell LC, Richman AR, Clawson K (2016) What do parents think about chromosomal microarray testing? A qualitative report from parents of children with ASD. Autism Research and Treatment.

33. King $M$, Bearman $P$ (2009) Diagnostic change and increased prevalence of autism. Int J Epidemiol 38: 12241234.

34. Van Naarden, Braun K, Christensen D, Doernberg N, Schieve L, et al. (2015) Trends in the prevalence of autism spectrum disorder, cerebral palsy, hearing loss, intellectual disability, and vision impairment. PLoS One 10: e0124120.

35. Watson MS, Mann MY, Lloyd-Puryear MA, Rinaldo P, Howell R (2006) Newborn screening: Toward a uniform screening panel and system. Pediatrics 117: 296-307.

36. Andermann A, Blancquaert I, Beachamp S, Dery V (2008) Revisiting Wilson and Jungner in the genomic age: $A$ review of screening criteria over the past 40 years. Bull World Health Organ 86: 317-319.

37. Friedman JM, Cornel MC, Goldenberg AJ, Lister KJ, Sénécal K, et al. (2017) Genomic newborn screening: public health policy considerations and recommendations. BMC Med Genomics 10: 9. 